\title{
Front-Loading Sputum Microscopy Services: An Opportunity to Optimise Smear-Based Case Detection of Tuberculosis in High Prevalence Countries
}

\author{
Andy Ramsay, ${ }^{1,2}$ Mohammed Ahmed Yassin,, ${ }^{1,3}$ Alexis Cambanis, ${ }^{1}$ \\ Susumu Hirao, ${ }^{1}$ Ahmad Almotawa, ${ }^{1}$ Mohamed Gammo, ${ }^{1}$ Lovett Lawson, ${ }^{4}$ \\ Izabel Arbide, ${ }^{5}$ Nasher Al-Aghbari, ${ }^{6}$ Najla Al-Sonboli, ${ }^{6}$ Jeevan Bahadur Sherchand, \\ Punita Gauchan, ${ }^{7}$ and Luis Eduardo Cuevas ${ }^{1}$ \\ ${ }^{1}$ Liverpool School of Tropical Medicine, Liverpool L3 5QA, UK \\ ${ }^{2}$ UNICEF/UNDP/World Bank/WHO Special Programme for Research and Training in Tropical Diseases, World Health Organization, \\ 1211 Geneva 27, Switzerland \\ ${ }^{3}$ TB and Leprosy Control Programme, Southern Region Health Bureau, P.O. Box 149, Awassa, Ethiopia \\ ${ }^{4}$ Zankli Medical Centre, P.O. Box 7745, Abuja, Nigeria \\ ${ }^{5}$ Bushullo Major Health Centre, P.O. Box 5, Awassa, Ethiopia \\ ${ }^{6}$ National Tuberculosis Institute, Sana'a, Yemen \\ ${ }^{7}$ Microbiology and Parasitology and Health Research Laboratory, Infectious and Tropical Disease Research Centre, \\ Institute of Medicine, Tribhuvan University, Kathmandu, Nepal P.O. Box 10404, Nepal \\ Correspondence should be addressed to Luis Eduardo Cuevas, lcuevas@liv.ac.uk
}

Received 14 October 2008; Revised 16 January 2009; Accepted 30 January 2009

Recommended by Marcel Tanner

Setting. Ethiopia, Nepal, Nigeria, and Yemen. Objective. To reduce the time to complete sputum microscopy. Design. Cross-sectional surveys enrolling 923 patients with chronic cough in the 4 countries and using similar protocols. Spot-morning-spot sputum specimens were collected. An additional sputum specimen (Xspot) was collected one hour after the first, and the yields of the first two or the three specimens collected as spot-morning-spot or spot-Xspot-morning were compared. Results. 216 patients had $\geq$ one positive smear. 210 (97\%) were identified by the spot-morning-spot, and 210 (97\%) were identified by the spot-Xspot-morning specimens, with 203 and 200 identified by the first 2 specimens of each approach, respectively. Neither difference was significant. Conclusions. The time to complete smear microscopy could be reduced.

Copyright (c) 2009 Andy Ramsay et al. This is an open access article distributed under the Creative Commons Attribution License, which permits unrestricted use, distribution, and reproduction in any medium, provided the original work is properly cited.

\section{Introduction}

New diagnostics for pulmonary tuberculosis (PTB) that are more sensitive than sputum smear microscopy and suitable for primary health care (PHC) services in low- and middleincome countries (LMICs) are urgently needed. Although several promising new diagnostics are under development, they are unlikely to become widely available at the lower levels of LMIC health services in the near future [1]. In the meantime, TB case detection must be improved through the optimal use of existing diagnostic tools. The optimisation of sputum microscopy services, often the only TB diagnostic services possible at PHC level in LMICs, is urgently needed [2-6]. Smear microscopy has several limitations, including poor sensitivity, being labour intensive, and requiring skilled microscopists. Furthermore, the need to collect serial sputum specimens over multiple patient visits results in a protracted diagnostic process with high rates of patient dropout $[7,8]$.

Recent studies examining the yield of serial sputum specimens, usually collected as spot-morning-spot, have reported that the majority of patients with smear-positive PTB are identified by the first two sputum specimens [2], and the World Health Organization (WHO) has recently changed 
its policy in this respect, reducing the minimum number of sputum specimens examined for each patient from three to two [9]. This will result in reduced laboratory workloads in many settings, with the potential of improving the quality of sputum microscopy [10]. Case detection may thus be expected to increase in locations where the number of new cases detected through improved microscopy quality exceeds the $2 \%$ to $5 \%$ of cases missed by not examining the third specimen [5].

The policy changes do not, however, specify the timing for the collection of the two specimens. If specimens were collected at the time of consultation (1st on-the-spot) and the morning of the following day (morning sample), the spotmorning specimens would still require a minimum of two visits, which is the minimum required by the spot-morningspot scheme currently used in most diagnostic centres of LMICs. In addition, the spot-morning and spot-morning-spot schemes still examine a substantial proportion of samples the second day of the diagnostic process. If the process could be "front-loaded", that is, if all or the majority of sputum collections were conducted the first day of the diagnostic process, this may reduce the number of visits required and reduce patient drop-out, particularly if results could be made available the same day.

This study describes the yield of a front-loaded diagnostic scheme, in which an additional on-the-spot specimen (referred here as the Xspot) is collected one hour after the first spot specimen. We hypothesised that the yield of this specimen is similar to the yield of other specimens collected on the spot, and that the overall yields of the spot-Xspotmorning and the standard spot-morning-spot schemes are similar. In addition, the study explores whether the first two specimens collected identify the majority of smear-positive patients.

\section{Materials and Methods}

Four separate studies were conducted in Ethiopia, Nepal, Nigeria, and Yemen using similar study designs. All individuals 15 years of age or older with cough for $\geq 3$ weeks were invited to participate and were enrolled consecutively at the time of presentation to the health services and after obtaining informed consent. The services in all four settings were busy outpatient clinics of district hospitals that were integrated with the National TB Control Programme of the country. Individuals either self-presented to these clinics or had been referred from peripheral health centres for assessment. Patients were requested to submit 3 sputum specimens as spot-morning-spot (the standard approach), and an additional specimen was collected one hour after the first (the Xspot) on the first day of consultation (Figure 1). All smears were stained using the hot Ziehl-Neelsen method, were read blindly by trained laboratory technicians, and graded using the WHO/IUATLD system. In accordance with the recent WHO policy changes, all smears with $\geq 1$ acid fast bacillus / 100 high power fields were considered positive. The main difference in study design between the four sites was that all morning specimens collected in Nepal and Yemen were cultured on solid egg-based media to allow for the calculation of sensitivity and specificity when considering culture as the reference standard. The four study sites had internal quality control procedures in place. External quality assessment (EQA) of smear microscopy was conducted by the Liverpool School of Tropical Medicine (LSTM), UK. For all 4 sites, there was $>98 \%$ agreement between the results of the study microscopists and those of the controllers at LSTM.

Data were analysed to describe the yield of single smears and the cumulative yields of the standard (spot-morningspot) versus the front-loaded (spot-Xspot-morning) schemes and of the two smear spot-morning versus the front-loaded spot-Xspot schemes. Proportions were described as percentages and $95 \%$ confidence intervals $(95 \% \mathrm{CI})$. A number of patients $(62,7 \%)$ did not have complete sets of sputum specimens for analysis. These patients were included in the analysis for the additional yield, and missing smears were considered as negative. As the schemes were not independent, comparisons of marginal proportions were made using matched McNemar tests. These included comparisons of the extra yield of the third sputum for each scheme; the yield of the "two" sample schemes and the comparison of the dropouts if patients were examined by the standard and frontloaded schemes. The extra yield of the third sputum for each scheme was summarised in $3 \times 3$ tables using the categories "positive on at least one of the first two samples", "positive on the third sample only", and "negative on all samples" and stratified by study setting. As this analysis required complete sets of data, the $62(7 \%)$ patients with missing sputum samples were excluded from this analysis. The yield for the "two" sample schemes was also compared with $2 \times 2$ tables with the indicators for each scheme being "positive on at least one of the two specimens" and "negative on both specimens". Patients with one of the two specimens missing $(36,4 \%)$ were classified as negative if the specimen available was negative. McNemar and Stuart MaxWell tests for marginal heterogeneity were used for comparison of the two and three specimen schemes, respectively, stratified by study site.

Partial results of the studies in Ethiopia and Nigeria, which described the yield of two smears collected as spot and Xspot in a single day, have been reported previously $[3,4]$. However, this report includes the yields of the spot-morningspot and spot-Xspot-morning schemes from these sites as well as the data from two additional sites conducting studies with similar design approaches.

Ethical approval for the study protocols was obtained from the research ethics committees of the Liverpool School of Tropical Medicine and the Institutional Review Boards of the participating institutions in Ethiopia, Nepal, Nigeria, and Yemen.

\section{Results}

A total of 923 consecutive patients were recruited. Of these, 243 were enrolled in Ethiopia, 206 in Nepal, 224 in Nigeria, and 250 in Yemen. Two hundred and sixteen $(23 \%)$ of the 923 patients had one or more positive smears. The 


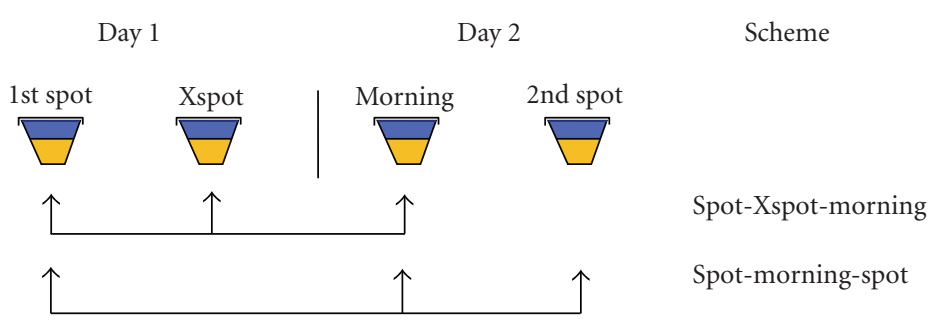

FIGURE 1: Sputum samples collected and analysed for each scheme.

TABLE 1: Incremental yield of serial smears collected as spot-morning-spot or spot-Xspot-morning specimens.

\begin{tabular}{|c|c|c|c|c|c|c|c|c|c|}
\hline \multirow{3}{*}{ Country } & \multirow{3}{*}{$N^{*}$} & \multirow{3}{*}{$\begin{array}{c}\geq 1 \text { pos. smear, } \\
N(\%)\end{array}$} & \multirow{3}{*}{$\begin{array}{c}\text { First spot pos. } \\
\quad N(\%)\end{array}$} & \multicolumn{3}{|c|}{ Standard approach } & \multicolumn{3}{|c|}{ Front-loaded approach } \\
\hline & & & & \multicolumn{2}{|c|}{ Incremental yield, $N(\%)$} & \multirow[t]{2}{*}{$N(\%)$ detected } & \multicolumn{2}{|c|}{ Incremental yield, $N(\%)$} & \multirow{2}{*}{$N(\%)$ detected } \\
\hline & & & & Morning & Second spot & & Xspot & Morning & \\
\hline Ethiopia & 243 & $52(21)$ & $45(87)$ & $4(8)$ & $2(4)$ & $51(98)$ & $4(8)$ & $3(6)$ & $52(100)$ \\
\hline Nepal & 206 & $55(25)$ & $39(71)$ & $7(13)$ & $5(9)$ & $51(93)$ & $5(9)$ & $6(11)$ & $50(91)$ \\
\hline Nigeria & 224 & $48(21)$ & $45(94)$ & $3(6)$ & 0 & $48(100)$ & $2(4)$ & $1(2)$ & $48(100)$ \\
\hline Yemen & 250 & $61(24)$ & $52(85)$ & $8(13)$ & 0 & $60(98)$ & $7(12)$ & $1(2)$ & $60(98)$ \\
\hline All & 923 & $216(23)$ & $181(84)$ & $22(10)$ & $7(3)$ & $210(97)$ & $18(8)$ & $11(5)$ & $210(97)$ \\
\hline
\end{tabular}

${ }^{*} N=$ number; pos = positive; Xspot = Extra spot collected one hour after the first spot sputum specimen.

first-spot, Xspot, morning, and second-spot specimens were graded positive in $181(20 \%), 186(20 \%), 185(20 \%)$, and $176(19 \%)$ patients, respectively, as shown in Table 1. In addition to the 181 patients identified by the first-spot, $22(10 \%)$ and $7(3 \%)$ additional patients were detected by the morning and second-spot specimens, respectively, resulting in a cumulative yield for the spot-morning-spot scheme of $210(23 \%)$ patients. In comparison, the Xspot and morning specimens of the front-loaded approach detected $18(8 \%)$ and $11(5 \%)$ additional patients, also resulting in a cumulative yield for the spot-Xspot-morning scheme of $210(23 \%)$ patients. The yield of the third smear was the same in both approaches, independently on whether this was thesecond spot or the morning specimen $(P>.5)$. A comparison of the yield of the third smear for samples collected using the standard (spot-morning-spot) and the front-loading (spot-Xspot-morning) approaches by country is shown in Table 2. Although there were a few discrepant results in the yield of the third specimen across the schemes, the direction of these discrepancies varied across study sites, and the discrepancies in each study site or combined were not statistically significant.

The first two (spot-morning) smears of the spot-morningspot approach identified 203 (97\%) smear-positive patients. These two smears, therefore, would have missed 7 (3\%, $95 \%$ CI $1 \%-6 \%$ ) of the cases identified by the three smears, as shown in Table 1. The first two (spot-Xspot) smears of the spot-spot-morning approach identified 199 (95\%, 91\%-97\%) smear-positive patients. These two spot smears, therefore, would have missed 11 cases $(5 \%, 95 \%$ CI 3\%-9\%) identified by the three smears. A matched comparison of the yield of two smears collected using the standard (spot-morning) and the front-loading (spot-Xspot) approaches stratified by country is shown in Table 3. Again, the proportion of cases missed by the spot-morning and the spot-spot smears is not statistically different.

Fifty-one (25\%) of the 206 patients in Nepal and 72 (29\%) of the 250 patients in Yemen had a positive TB culture. Thirty-nine and 37 of the 51 culture-positive patients in Nepal were identified by the standard and the front-loaded schemes, resulting in a sensitivity of $76 \%$ [95\%CI $63 \%-$ $87 \%]$ and $73 \%$ [59\%-83\%], respectively. In Yemen, 51 and 52 of the 72 culture-positive patients were identified by the standard and front-loaded schemes, respectively, (sensitivity $71 \%[60 \%-80 \%]$ and $72 \%[61 \%-82 \%])$. There were no statistical differences between the sensitivities of the standard and front-loaded approaches.

The sensitivity of two smears collected as spot-morning would be $69 \%$ (35 of 51 culture positive patients) in Nepal and $71 \%$ (51 of 72) in Yemen. In comparison, the sensitivity of the two smears collected as spot-Xspot would be 67\% (34 of $51)$ in Nepal and 67\% (48 of 72) in Yemen. These differences are not statistically significant $(P>.4$ for both).

\section{Discussion}

Much progress has been made in recent years in the development of new diagnostics for TB. However, few of the newer diagnostic technologies are suitable for use outside of reference laboratories in the public health services of LMICs. TB control in resource-poor, high-prevalence settings, therefore, will continue to rely upon sputum smear microscopy until frontline services gain access to the new technologies. Tuberculosis affects disproportionately indigent populations who seek health services in areas with 
TABLE 2: Yield of the third smear for samples collected using the standard (spot-morning-spot) and the front-loading (spot-spot-morning) approaches by country. 62 patients ( 15 from Yemen, 44 from Nepal, and 3 from Ethiopia) were excluded as they did not submit the third specimen.

\begin{tabular}{|c|c|c|c|c|}
\hline \multirow{2}{*}{$\begin{array}{l}\text { Standard approach } \\
\text { Yemen }(N=235)\end{array}$} & \multicolumn{3}{|c|}{ Front-loading approach } & \multirow[t]{2}{*}{$P^{*}$} \\
\hline & Pos on 1st two smears & Only 3rd smear pos & All negative & \\
\hline Pos on 1st two smears & 56 & 4 & 0 & 14 \\
\hline Only 3rd smear pos & 0 & 0 & 0 & \\
\hline All negative & 0 & 0 & 175 & \\
\hline \multicolumn{5}{|l|}{$\operatorname{Nepal}(N=162)$} \\
\hline Pos on 1st two smears & 40 & 6 & 0 & \multirow{3}{*}{.76} \\
\hline Pos on the 3 rd smear & 3 & 0 & 5 & \\
\hline Negative in all & 3 & 0 & 105 & \\
\hline \multicolumn{5}{|l|}{ Nigeria $(N=224)$} \\
\hline Pos on 1st two smears & 47 & 1 & 0 & \multirow{3}{*}{.61} \\
\hline Pos on the 3rd smear & 0 & 0 & 0 & \\
\hline Negative in all & 0 & 0 & 176 & \\
\hline \multicolumn{5}{|l|}{ Ethiopia $(N=240)$} \\
\hline Pos on 1st two smears & 46 & 3 & 0 & \multirow{3}{*}{.55} \\
\hline Pos on the 3rd smear & 2 & 0 & 0 & \\
\hline Negative in all & 1 & 0 & 188 & \\
\hline \multicolumn{5}{|l|}{ All sites $(N=861)$} \\
\hline Pos on 1st two smears & 189 & 14 & 0 & \multirow{3}{*}{.58} \\
\hline Pos on the 3rd smear & 5 & 0 & 5 & \\
\hline Negative in all & 4 & 0 & 644 & \\
\hline
\end{tabular}

* Stuart-MaxWell test, (Marginal Heterogeneity).

TABLE 3: Yield of two smears collected using the standard (spot-morning) and the frontloading (spot-Xspot) approaches by country.

\begin{tabular}{|c|c|c|c|c|}
\hline \multirow{2}{*}{$\begin{array}{l}\text { Standard approach } \\
\text { Yemen }\end{array}$} & \multicolumn{3}{|c|}{ Frontloading approach } & \multirow[t]{2}{*}{$P^{*}$} \\
\hline & Positive & Negative & All & \\
\hline Positive & 56 & 4 & 60 & 18 \\
\hline Negative & 1 & 189 & 190 & \\
\hline All & 57 & 193 & 250 & \\
\hline \multicolumn{5}{|l|}{ Nepal } \\
\hline Positive & 40 & 6 & 46 & \multirow{3}{*}{.53} \\
\hline Negative & 4 & 156 & 160 & \\
\hline All & 44 & 162 & 206 & \\
\hline \multicolumn{5}{|l|}{ Nigeria } \\
\hline Positive & 47 & 1 & 48 & \multirow{3}{*}{.32} \\
\hline Negative & 0 & 176 & 176 & \\
\hline All & 47 & 177 & 224 & \\
\hline \multicolumn{5}{|l|}{ Ethiopia } \\
\hline Positive & 46 & 3 & 49 & \multirow{3}{*}{1.0} \\
\hline Negative & 3 & 191 & 194 & \\
\hline All & 49 & 194 & 243 & \\
\hline \multicolumn{5}{|l|}{ All sites } \\
\hline Positive & 189 & 14 & 203 & \multirow{3}{*}{.58} \\
\hline Negative & 8 & 712 & 720 & \\
\hline All & 197 & 726 & 923 & \\
\hline
\end{tabular}

\footnotetext{
* McNemar test.
} 
limited resources [11], and the costs incurred by patients can be prohibitively high, even when services are provided free of charge [12]. Individuals need to attend health facilities on several occasions to see a clinician, submit sputum specimens, receive results, and be put on treatment, and these visits may incur loss of earnings and require repeated travel, purchase of food, and accommodation [13]. A significant proportion of people undergoing investigations for TB dropout of the smear diagnostic pathway, and these patients are more likely to be the poor. Although few studies report the proportion of patients who drop out during the diagnosis of TB in LMIC, $13 \%$ of TB suspects in India [14], 15\% and $37 \%$ of smear-positive patients in rural [8], and urban Malawi [15] dropout of the process, and unusually, the diagnostic dropout rate can be as high as 95\% [16]. Failing to complete the diagnosis, therefore, is a major obstacle to access treatment in these settings [8].

There is increasing interest in equity in health services, as typified by the interim report of the WHO Commission on Social Determinants of Health. Primary health care, once again, plays a central role in WHO's current agenda, and the development of equitable diagnostic services is, therefore, paramount to access treatment [17]. The investigation of suspected PTB in many low-prevalence countries is based on the examination of serial morning specimens because these specimens have been associated with a significant additional yield of patients. The proportion of patients who drop out in these settings, however, is insignificant, and the spotmorning-spot scheme was developed in the 1950s and 60s in response to the need to reduce the number of visits in high-prevalence areas, where patients often abandoned the diagnostic process. Although it was widely accepted that overnight specimens were more likely to contain more bacilli, it was also acknowledged that in less favourable circumstances, it was more practical to obtain specimens at the time the patient was attending the service [18]. Two of the morning specimens were replaced by spot specimens, and shorter schemes that required a reduced number of visits were developed. Although this was a remarkable improvement, the continued need for multiple visits was still a hindrance for many patients.

It is now recognised that the high bacillary threshold for defining a smear as positive and the requirement to obtain at least one confirmatory smear have unnecessarily reduced the sensitivity of the test in the detection of smear positive cases. These requirements also resulted in many laboratory services being overwhelmed and leaving insufficient time for the examination of smears. The recent WHO policy changes reduce the smear microscopy thresholds [9] and the minimum number of specimens to be examined. These changes will reduce workload, particularly important in areas, where skilled human resources are limited, potentially increasing case detection through allowing more time to examine the smears [10]. These policy changes might be associated with larger gains in case detection if the timing of sputum specimen collection and examination were more convenient for patients, particularly poor patients, and help to reduce drop-out.
This study shows that the spot-spot-morning and spotmorning-spot schemes have similar yields. This indicates that front-loaded TB diagnostic services (whether based on the examination of two or three specimens) are feasible and would not be associated with significantly less yield than the equivalent standard approach. Both two-smear strategies would miss about $3 \%-5 \%$ of the patients identified by the three-specimen strategy, as suggested by a previous systematic review $[5,19]$. These losses are likely to be compensated by increased quality of microscopy and lower drop-out rates of patients. In addition, programmes where $\geq 10 \%$ of patients fail to return for the second day of diagnosis would identify similar numbers using 2 smears the first day of consultation than 2 or 3 smears collected over two or more days. Countries adopting two-smear schemes, therefore, may consider collecting and examining the specimens in a single day to shorten the time required for diagnosis.

The development of diagnostic approaches that are responsive to the needs of the population may be feasible, and larger studies are urgently required to validate the findings of this study under operational conditions. If the findings of this study are confirmed, smear microscopy services should be front loaded in the interests of equity and improved TB control.

\section{Summary}

The diagnosis of tuberculosis in high-burden settings relies on sputum smear microscopy and requires multiple patients' visits to the health facilities. This approach could be improved if most specimens were collected the first day of consultation. This study reports the smear microscopy findings of 923 adults with chronic cough participating in four cross-sectional surveysin Ethiopia, Nepal, Nigeria, and Yemen. Sputum specimens were collected as spotmorning-spot plus one additional specimen one hour after the first spot (X-spot). The yield of two (spot-Xspot or spot-morning) or three (spot-morning-spot or spot-X-spotmorning) specimens was compared. 216 patients had $\geq$ one positive smear. Of these, $210(97 \%)$ were identified by the spot-morning-spot, and 210 (97\%) were identified by the spot-Xspot-morning specimens. Spot-morning identified 203 and spot-Xspot specimens 200 patients, respectively, $(P>.1)$. The time, number of visits and patients' costs to complete smear microscopy could be reduced by frontloading the collection of sputum specimens.

\section{Acknowledgments}

The authors are grateful to Professors Martin Borgdoff and Paul Klatser for their review and useful criticism of the manuscript prior to submission. The studies were funded by study scholarships for Drs Cambanis, Hirao, Gammo, and Almotawa. The authors do not have a commercial or other association that might pose a conflict of interest. Preliminary data was shown as an oral presentation, WHO workshop on new diagnostics, XXXVII Union Conference "Feasibility and 
sensitivity of a one day smear microscopy approach for the diagnosis of TB," November 2006, Paris, France.

\section{References}

[1] M. Guillerm, M. Usdin, and J. Arkinstall, "Tuberculosis diagnosis and drug sensitivity testing: an overview of the current diagnostic pipeline," 2006, Médicins Sans Frontieres.

[2] M. Bonnet, A. Ramsay, L. Gagnidze, W. Githui, P. J. Guerin, and F. Varaine, "Reducing the number of sputum samples examined and thresholds for positivity: an opportunity to optimise smear microscopy," International Journal of Tuberculosis and Lung Disease, vol. 11, no. 9, pp. 953-958, 2007.

[3] A. Cambanis, M. A. Yassin, A. Ramsay, S. B. Squire, I. Arbide, and L. E. Cuevas, "A one-day method for the diagnosis of pulmonary tuberculosis in rural Ethiopia," International Journal of Tuberculosis and Lung Disease, vol. 10, no. 2, pp. 230-232, 2006.

[4] S. Hirao, M. A. Yassin, H. G. Khamofu, et al., "Same-day smears in the diagnosis of tuberculosis," Tropical Medicine and International Health, vol. 12, no. 12, pp. 1459-1463, 2007.

[5] S. R. Mase, A. Ramsay, V. Ng, et al., "Yield of serial sputum specimen examinations in the diagnosis of pulmonary tuberculosis: a systematic review," International Journal of Tuberculosis and Lung Disease, vol. 11, no. 5, pp. 485-495, 2007.

[6] M. S. Khan, O. Dar, C. Sismanidis, K. Shah, and P. GodfreyFaussett, "Improvement of tuberculosis case detection and reduction of discrepancies between men and women by simple sputum-submission instructions: a pragmatic randomised controlled trial," The Lancet, vol. 369, no. 9577, pp. 1955-1960, 2007.

[7] E. Keeler, M. D. Perkins, P. Small, et al., "Reducing the global burden of tuberculosis: the contribution of improved diagnostics," Nature, vol. 444, supplement 1, pp. 49-57, 2006.

[8] S. B. Squire, A. K. Belaye, A. Kashoti, et al., "'Lost' smearpositive pulmonary tuberculosis cases: where are they and why did we lose them?" International Journal of Tuberculosis and Lung Disease, vol. 9, no. 1, pp. 25-31, 2005.

[9] WHO, "New WHO policies," December 2007, http://www .who.int/tb/dots/laboratory/policy/en/index.html.

[10] A. Cambanis, A. Ramsay, V. Wirkom, E. Tata, and L. E. Cuevas, "Investing time in microscopy: an opportunity to optimise smear-based case detection of tuberculosis," International Journal of Tuberculosis and Lung Disease, vol. 11, no. 1, pp. 4045, 2007.

[11] I. Bates, C. Fenton, J. Gruber, et al., "Vulnerability to malaria, tuberculosis, and HIV/AIDS infection and disease-part 1: determinants operating at individual and household level," The Lancet Infectious Diseases, vol. 4, no. 5, pp. 267-277, 2004.

[12] J. R. Kemp, G. Mann, B. N. Simwaka, F. M. L. Salaniponi, and S. B. Squire, "Can Malawi's poor afford free tuberculosis services? Patient and household costs associated with a tuberculosis diagnosis in Lilongwe," Bulletin of the World Health Organization, vol. 85, no. 8, pp. 580-585, 2007.

[13] A. Cambanis, M. A. Yassin, A. Ramsay, S. B. Squire, I. Arbide, and L. E. Cuevas, "Rural poverty and delayed presentation to tuberculosis services in Ethiopia," Tropical Medicine and International Health, vol. 10, no. 4, pp. 330-335, 2005.

[14] V. Chandraseekaran, R. Ramachandran, J. Cunningham, et al., "Factors leading to tuberculosis diagnostic drop-out and delayed treatment initiation in Chennai, India," International
Journal of Tuberculosis and Lung Disease, vol. 9, no. S1, p. S172, 2005.

[15] J. Kemp, S. B. Squire, I. K. Nyirenda, and F. M. L. Salaniponi, "Is tuberculosis diagnosis a barrier to care?" Transactions of the Royal Society of Tropical Medicine and Hygiene, vol. 90, p. 472, 1996.

[16] A. Nota, H. Ayles, M. Perkins, and J. A. Cunningham, "Factors leading to tuberculosis diagnostic drop-out and delayed treatment initiation in urban Lusaka," International Journal of Tuberculosis and Lung Disease, vol. 9, no. 11, p. S305, 2005.

[17] WHO, "Interim Report of WHO Commission on Social Determinants of Health," 2007.

[18] R. H. Andrews and S. Radhakrishna, "A comparison of two methods of sputum collection in the diagnosis of pulmonary tuberculosis," Tubercle, vol. 40, no. 3, pp. 155-162, 1959.

[19] K. R. Steingart, A. Ramsay, and M. Pai, "Optimizing sputum smear microscopy for the diagnosis of pulmonary tuberculosis," Expert Review of Anti-Infective Therapy, vol. 5, no. 3, pp. 327-331, 2007. 


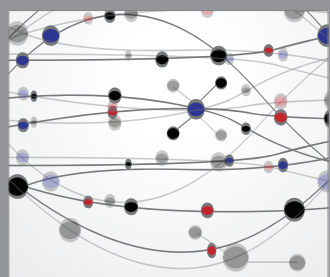

The Scientific World Journal
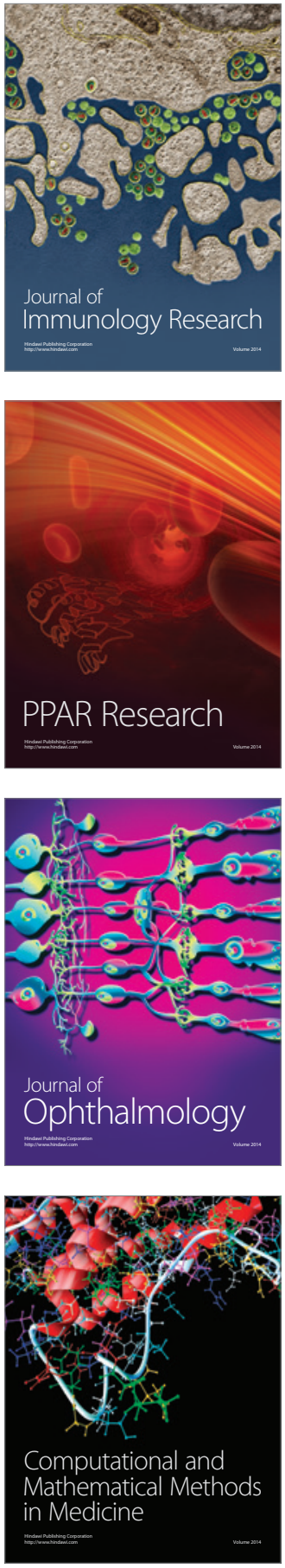

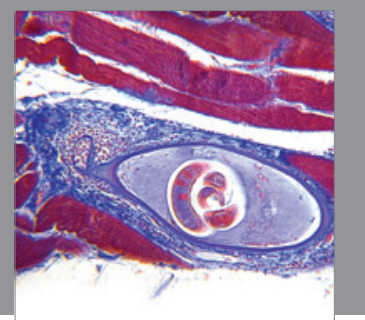

Gastroenterology

Research and Practice
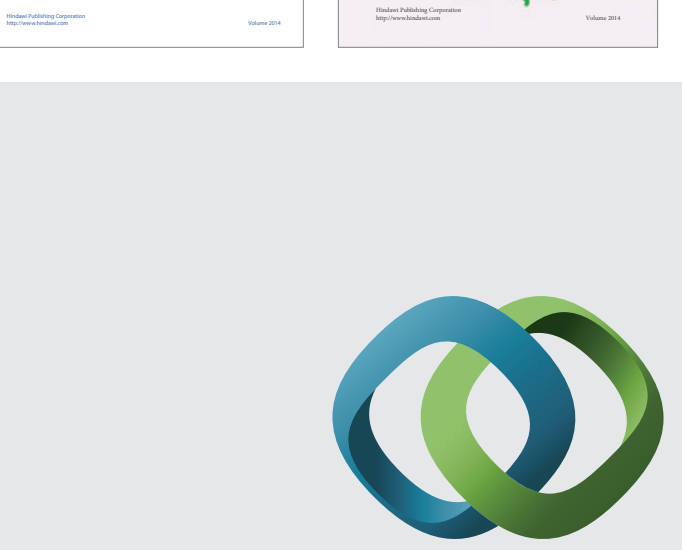

\section{Hindawi}

Submit your manuscripts at

http://www.hindawi.com
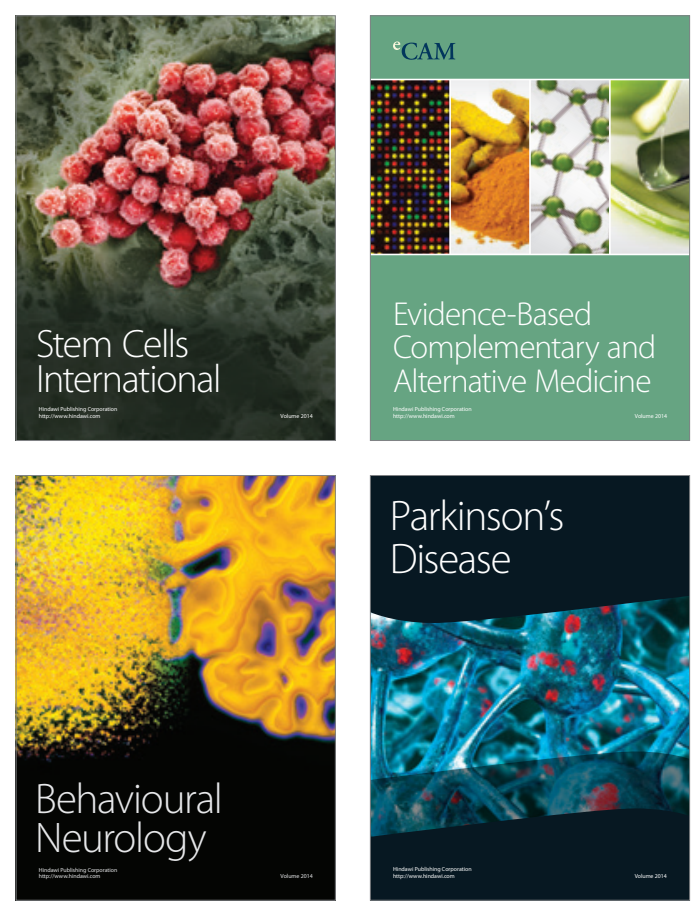

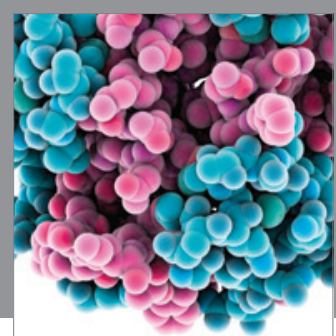

Journal of
Diabetes Research

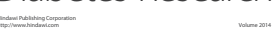

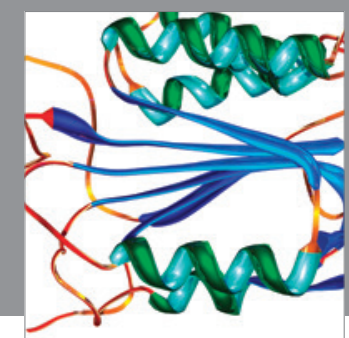

Disease Markers
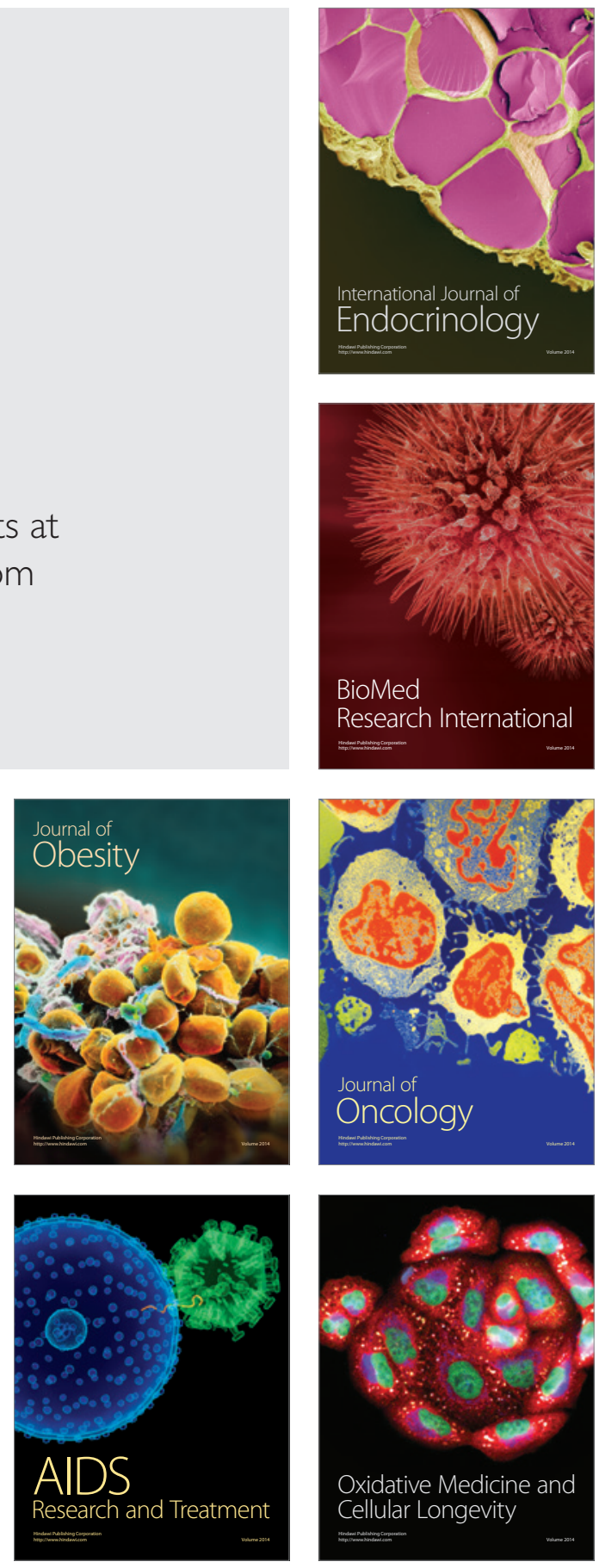